\title{
Low frequency eigenmodes of thin anisotropic current sheets and Cluster observations
}

\author{
L. M. Zelenyi ${ }^{1}$, A. V. Artemyev $^{1}$, A. A. Petrukovich ${ }^{1}$, R. Nakamura ${ }^{4}$, H. V. Malova ${ }^{1,2}$, and V. Y. Popov ${ }^{1,3}$ \\ ${ }^{1}$ Space Research Institute, Russian Academy of Sciences, Moscow, Russia \\ ${ }^{2}$ Nuclear Physics Institute, Moscow State University, Russia \\ ${ }^{3}$ Faculty of Physics, Moscow State University, Russia \\ ${ }^{4}$ Space Research Institute, Austrian Academy of Sciences, Graz, Austria
}

Received: 14 August 2008 - Revised: 2 December 2008 - Accepted: 9 December 2008 - Published: 19 February 2009

\begin{abstract}
The eigenmodes of low frequency perturbations of thin anisotropic current sheets with a finite value of the normal magnetic field, are investigated in this paper. It is shown that two possible polarizations of symmetric and asymmetric modes (sausage and kink) exist where the growth rate of instabilities is positive. In addition, we demonstrate that a tearing instability might have a positive growth rate in thin anisotropic current sheets. The class of relatively fast wavy flapping oscillations observed by Cluster is described. The main direction of wave motion coincides with the direction of the current and the typical velocity of this motion is comparable with the plasma drift velocity in the current sheet. The comparison of these characteristics with theoretical predictions of the model of anisotropic thin current sheets, demonstrates that, in principle, the theory adequately describes the observations.
\end{abstract}

Keywords. Magnetospheric physics (Magnetotail) - Space plasma physics (Numerical simulation)

\section{Introduction}

The numerous attempts to describe current sheets (CSs) in the Earth's magnetosphere and to investigate their stability led to the development of a variety of models of CS. The simplest CS models are the one of Harris (1962) and its 2-D generalization by Kan (1973). These models are usually used to describe thick isotropic CS equilibrium. Another group of models, taking into account the anisotropy of ion distribu-

Correspondence to: A. V. Artemyev (ante0226@gmail.com) tions and/or quasi-adiabaticity of ion motion in thin current sheets (where ion gyroradii are about CS thickess), was recently developed (Zelenyi et al., 2004; Sitnov et al., 2006). Earlier, the theory of stability was applied to study eigenmodes of the Harris CS, therefore the tearing (Coppi et al., 1966; Schindler, 1974; Galeev and Zelenyi, 1976; Pellat et al., 1991; Sitnov et al., 1997), kink (Daughton, 1999) and sausage modes (Lapenta and Brackbill, 1997; Buechner and Kuska, 1999) were investigated in detail. It was shown that the Harris CS is completely stable under tearing perturbations in the presence of a finite value of a normal magnetic component (Pellat et al., 1991) because of a strong stabilizing effect of the magnetized electrons. This situation is different if one takes into account some additional effects, for example, the presence of transient electrons in the plasma population (Sitnov et al., 1997).

It was shown, in newly obtained spacecraft observations, that the properties of relatively thin CSs are completely different from Harris ones (Nakamura et al., 2006; Runov et al., 2006) and a more adequate approximation might be achieved in a frame of recent CS models (Sitnov et al., 2006; Baumjohann et al., 2007; Artemyev et al., 2008).

Contrary to well-known isotropic models, the general theory of anisotropic thin CS (TCS) instabilities, which might be useful to compare the typical temporal and spatial characteristics of observed CSs, has not been developed yet. Very few successful attempts to compare the experimental data of CS dynamics with theoretical results have been made in the last years (Volwerk et al., 2003; Golovchanskaya et al., 2005; Erkaev et al., 2008; Saito et al., 2008). In this article, we present our first results devoted to the further development of the general theory of oblique instabilities (of tearing, kink, sausage and oblique eigenmodes) in anisotropic TCSs

Published by Copernicus Publications on behalf of the European Geosciences Union. 
(Zelenyi et al., 2004). Theoretical predictions are compared with experimental Cluster data. We demonstrate here that, contrary to Harris-like CSs, which are always stable under tearing perturbation in the presence of a finite magnetic component $B_{z}$ (Pellat et al., 1991), the tearing mode in TCSs might be unstable (Zelenyi et al., 2008), and its growth depends sensitively on the propagation angle (we use here the Geocentric Solar Magnetospheric coordinate system). Other TCS modes (e.g. kink and sausage), which propagate perpendicularly to the magnetic field, might also develop in anisotropic CSs with $B_{z} \neq 0$. The values of their growth rates are comparable.

\section{TCS model and essential parameters}

To investigate the CS instabilities, we have used the model of anisotropic TCS equilibrium (Zelenyi et al., 2004, 2006, and references therein). In this model, the ion component is described by a bi-Maxwellian distribution function (at the edges of the CS) that can be rewritten as a function of two integrals of motion: the particle energy and the adiabatic invariant of motion $I_{z}=(\mathrm{m} / 2 \pi) \oint v_{z} d z$ (the last one is approximately conserved during particle motion). The key free parameter of the model is $\varepsilon$, the ratio of the ion thermal velocity to the bulk plasma velocity along the field lines at the edges of the CS. This parameter determines the anisotropy of ion velocity distribution in the CS and controls the thickness of the CS $L_{C S}$ itself (Zelenyi et al., 2004). The electron component is defined in a flow approximation by the parameter $\tau=T_{i} / T_{e}$ (ratio of ion to electron temperatures). The parameter that controls the magnetic topology of the system is $b_{n}=B_{z} / B_{0}$ ( $B_{z}$ is the normal component of the magnetic field; $B_{0}$ is the magnetic field at the edges of the CS). The important characteristics of TCSs, principally different from isotropic CS models, are the shear of bulk velocity in the central region of the CS $j_{y}(z) /(e n(z)) \neq$ const and the embedding of the CS inside a much thicker plasma sheet (at the edges of the CS where $j_{y}=0$, the plasma density $n \neq 0$ ). Therefore, the excess of a free energy in TCS could be larger in comparison with isotropic CSs and, as a result, the growth rate of CS instabilities might be substantially higher (Zelenyi et al., 2008).

\section{Stability of TCSs and the energy principle}

In this paper, the stability of perturbations of vector potential in the form of oblique plane waves $\boldsymbol{A}_{1}=\widehat{\mathbf{A}}_{1}(z) \exp \left\{i k_{x} x+i k_{y} y-i \omega t\right\}$ in the reversed magnetic field $B_{x}$ with $B_{z} \neq 0$ is investigated. The electrons are assumed to be totally magnetized (Schindler, 1974). In this case, a strong stabilizing effect of the electron compressibility should be taken into account (Galeev and Zelenyi, 1976; Pellat et al., 1991; Schindler, 2006). On the temporal scale of the growth of instability, the ion motion is faintly perturbed by their oscillations in the $B_{z}$ magnetic field (Schindler, 1974) and the main ion contribution is due to Landau resonance with the waves.

To obtain the energy balance of these perturbations, the energy principle is used in its general form (Pellat et al., 1991; Schindler, 2006; Zelenyi et al., 2008). One can present the energy balance of a perturbed quasi-neutral system as:

$W=\int \frac{\boldsymbol{B}^{2}}{8 \pi} d^{3} r+\sum_{j} \int \frac{m_{j} \boldsymbol{v}^{2}}{2} f_{j} d^{3} r d^{3} v$

The first order of the perturbation of velocity distribution function $f_{1 j}$ might be found on the base of the linearized Vlasov equation:

$$
\begin{aligned}
& f_{1 j}-\frac{q_{j}}{m_{j} c} f_{0 j} \boldsymbol{S}_{j} \boldsymbol{A}_{1}=-\frac{q_{j}}{m_{j} c} f_{0 j} \\
& \int_{0}^{t}\left\{\boldsymbol{A}_{1} \overline{\boldsymbol{S}}_{j}+\left(-c \nabla \varphi_{1}+\nabla\left(\boldsymbol{v} A_{1}\right)\right) \boldsymbol{S}_{j}\right\} d t^{\prime}=f_{1 j}^{\mathrm{res}}
\end{aligned}
$$

where $\boldsymbol{S}_{j}=f_{0 j}^{-1}\left(\partial f_{0 j} / \partial \boldsymbol{v}\right)$ and $\overline{\boldsymbol{S}}_{j} \boldsymbol{e}_{\alpha}=\boldsymbol{v}\left(\partial\left(\boldsymbol{S}_{j} \boldsymbol{e}_{\alpha}\right) / \partial \boldsymbol{r}\right)$ $+\left(q_{j} / c m_{j}\right)\left(\boldsymbol{E}_{0} c+\left[\boldsymbol{v} \times \boldsymbol{B}_{0}\right]\right)\left(\partial\left(\boldsymbol{S}_{j} \boldsymbol{e}_{\alpha}\right) / \partial \boldsymbol{v}\right)$.

One can write the second order of perturbed energy $W^{(2)}$ as a function of $\boldsymbol{A}_{1}: \tilde{f}_{1 j}=f_{1 j}-\left(\partial f_{1 j} / \partial \boldsymbol{A}_{0}\right) \boldsymbol{A}_{1}-f_{1 j}^{\text {res }}$ :

$$
\begin{aligned}
W^{(2)}= & \int \frac{\boldsymbol{B}_{1}^{2}}{8 \pi} d \boldsymbol{r}-\frac{1}{2} \sum_{j} \int \frac{\tilde{f}_{1 j}^{2}}{\partial f_{0 j} / \partial H_{0 j}} d \boldsymbol{r} d \boldsymbol{p} \\
& -\sum_{j} \frac{1}{m_{j}} \int\left(\boldsymbol{p}-\frac{q_{j}}{c} \boldsymbol{A}_{0}\right) \frac{q_{j}}{c} \boldsymbol{A}_{1} f_{1 j} d \boldsymbol{r} d \boldsymbol{p} \\
& +\sum_{j} \frac{1}{2 m_{j}} \frac{q_{j}^{2}}{c^{2}} \int \boldsymbol{A}_{1}^{2} f_{0 j} d \boldsymbol{r} d \boldsymbol{p}
\end{aligned}
$$

Now, one should take into account that the zero order of the ion velocity distribution $f_{0 i}=f_{0 i}\left(H_{0 i}, I_{z}\right)$ depends on two integrals of motion: the exact one $H_{0 i}$ and the approximate one $I_{z}$, which allows us to rewrite the expression for the adiabatic invariant $I_{z}$ using the ion generalized momentum $P_{y i}$ of particle motion (Zelenyi et al., 2004) as:

$$
\begin{aligned}
I_{z}=\frac{2 m_{j}}{\pi} \int_{z_{0}}^{z_{1}}\left(v_{z}^{2}+v_{y}^{2}+2 q_{j} m_{j}^{-1}\left\{\varphi(z)-\varphi\left(z^{\prime}\right)\right\}\right. \\
\left.-\left\{v_{y}-\frac{q_{j}}{m_{j} c}\left(A_{0 y}\left(z^{\prime}\right)-A_{0 y}(z)\right)\right\}^{2}\right)^{1 / 2} d z^{\prime}
\end{aligned}
$$

Taking into account the approximate conservation of $I_{z}$ along the particle trajectory in the CS, Eq. (3) can be rewritten in a new form:

$$
\begin{aligned}
W^{(2)}= & \int \frac{\boldsymbol{B}_{1}^{2}}{8 \pi} d \boldsymbol{r}-\frac{1}{2} \sum_{j} \int \frac{\tilde{f}_{1 j}^{2}}{\partial f_{0 j} / \partial H_{0 j}} d \boldsymbol{r} d \boldsymbol{p} \\
& -\frac{1}{2 c} \int \frac{\partial \boldsymbol{j}_{0}}{\partial \boldsymbol{A}_{0}} \boldsymbol{A}_{1}^{2} d \boldsymbol{r}-\frac{1}{2 c} \int \boldsymbol{j}^{r e s} \boldsymbol{A}_{1} d \boldsymbol{r}
\end{aligned}
$$


Here, $\boldsymbol{j}^{\text {res }}=e \int \boldsymbol{v} f_{1 i}^{\text {res }} d \boldsymbol{v}$ is the density of the perturbed current due to resonance ion-wave interaction. The minimization of the functional $W^{(2)}\left(\boldsymbol{A}_{1}\right)$ in Eq. (5) allows us to find and investigate different CS perturbations. To achieve this aim, one should present $\tilde{f}_{1 j}$ as a function of the perturbation of vector potential $\boldsymbol{A}_{1}$, taking into account two properties: first, the quasi-neutrality of plasma $\sum_{j} q_{j} \int f_{1 j} d^{3} v=0$; second, the electron magnetization in the $B_{z}$ magnetic field, i.e. $n_{1 e} \approx n_{0}\left(B_{1 z} / B_{z}\right)$. Using Schwartz inequality (see Zelenyi et al., 2008), one can rewrite Eq. (5) in the following form:

$$
\begin{aligned}
8 \pi W^{(2)}= & \int\left\{\boldsymbol{B}_{1}^{2}+8 \pi p_{0}\left(k_{x} \boldsymbol{A}_{1} \boldsymbol{e}_{y} / B_{z}\right)^{2}\right. \\
& \left.-4 \pi c^{-1}\left(\partial \boldsymbol{j}_{0} / \partial \boldsymbol{A}_{0}\right) \boldsymbol{A}_{1}^{2}-4 \pi c^{-1} \boldsymbol{j}^{r e s} \boldsymbol{A}_{1}\right\} d \boldsymbol{r}(6)
\end{aligned}
$$

where $p_{0}$ is the total unperturbed kinetic pressure. The value of a free energy $\sim \partial \boldsymbol{j}_{0} / \partial \boldsymbol{A}_{0}$ in Eq. (6) is substantially different from the one in a Harris-like CS, where the derivative $\partial \boldsymbol{j}_{0} / \partial \boldsymbol{A}_{0} \sim \boldsymbol{j}_{0}$ is due to the constancy of the drift plasma velocity everywhere in the CS. In our case, this derivative has another form, i.e. $\partial \boldsymbol{j}_{0} / \partial \boldsymbol{A}_{0} \sim \boldsymbol{j}_{0} F(z)$, where $F(z)$ has a very strong maximum in the very centre of the CS. Also, one can see that the functional $W^{(2)}$ is dependent only on the perturbation of a vector potential. The corresponding dispersion ratio could easily be obtained if one accomplished a minimization of a functional $W^{(2)}\left(\boldsymbol{A}_{1}\right)$ under the condition $\delta W^{(2)} / \delta \boldsymbol{A}_{1}=0$.

\section{Numerical results}

We used the following parameters to investigate instabilities: $k=\sqrt{k_{x}^{2}+k_{y}^{2}}$ (the magnitude of the wave vector); $\theta=\arctan \left(k_{y} / k_{x}\right)$ (the angle of wave propagation). Also, we have taken into account the Coulomb gauge condition for the perturbed vector potential, i.e. $\operatorname{div} \boldsymbol{A}_{1}=0$. Now, one should consider two different polarizations of the perturbed vector potential $\boldsymbol{A}_{1}$. First, polarization might be presented in the form $\boldsymbol{A}_{1}=A_{1 x} \boldsymbol{e}_{x}+A_{1 y} \boldsymbol{e}_{y}$ (Galeev and Zelenyi, 1976; Silin et al., 2002). The Coulomb gauge imposes the following condition related to the components of the perturbed vector potential: $A_{1 x} \cos \theta+A_{1 y} \sin \theta=0$. This perturbation is suppressed at $\theta \rightarrow \pi / 2$. Perturbation with another polarization $\boldsymbol{A}_{1}=A_{1 y} \boldsymbol{e}_{y}+A_{1 z} \boldsymbol{e}_{z}$ (Lapenta and Brackbill, 1997) might grow at $\theta=\pi / 2$. In this paper, we consider the characteristics of both kinds of polarization.

According to the paper by Dobrowolny (1968), the width of the region of ion resonance interaction with growing waves $U_{i}(z)(i=1,2,3, \ldots)$ could be found from the conditions $U_{i}(z)=1$, if $\rho_{i}(z)<z$ and $U_{i}=0$, if $\rho_{i}(z)>z$ (where $\left.\rho_{i}(z)=v_{T i} / \omega_{i} b_{x}(z), \omega_{i}=e B_{x}(\infty) / m_{i} c, b_{x}(z)=B_{x} / B_{0}\right)$. We have taken into account only ion resonant currents because, in the presence of the normal component of the magnetic field $B_{z}$, the electron population becomes magnetized; consequently, it could not contribute to the resonant interaction (Schindler, 1974).

For the polarization of a vector potential $\boldsymbol{A}_{1}$ in the form $\boldsymbol{A}_{1}=A_{1 x} \boldsymbol{e}_{x}+A_{1 y} \boldsymbol{e}_{y}$, it is more convenient to consider the simplified equation for its value $A_{1}=\sqrt{A_{1 x}^{2}+A_{1 y}^{2}}$ instead of the system of two equations for each of these components. This is possible because $A_{1 x}$ and $A_{1 y}$ are linearly coupled (i.e. $A_{1 x}=-A_{1 y} \tan \theta$ ) by the Coulomb gauge. Therefore, one can rewrite the equation $\delta W^{(2)} / \delta \boldsymbol{A}_{1}=0$ in the following form:

$$
\begin{aligned}
d^{2} A_{1} / d z^{2}- & \left\{k^{2}\left(1+4 \pi p_{0} B_{z}^{-2} \cos ^{4} \theta\right)\right. \\
& \left.-4 \pi c^{-1}\left(\partial j_{y} / \partial A_{0}\right) \cos ^{2} \theta\right\} A_{1} \\
= & -j^{\mathrm{res}}\left(z, \theta, A_{1}, t\right)
\end{aligned}
$$

The current of resonant interaction $j^{\text {res }}\left(z, \theta, A_{1}, t\right)$ can be obtained as the integral $\int v_{y} f_{1 i}^{\text {res }} d^{3} v$. For another kind of polarization $\boldsymbol{A}_{1}=A_{1 y} \boldsymbol{e}_{y}+A_{1 z} \boldsymbol{e}_{z}$, one should solve the single equation for $A_{1 y}$ as it was done by Lapenta and Brackbill (1997) for the sausage mode $(\theta=\pi / 2)$. Contrary to previous works, we considered the perturbations propagating at arbitrary angles in the CS plane $(\theta \in[0, \pi / 2])$ :

$$
\begin{aligned}
d^{2} A_{1 y} / d z^{2}- & \left\{k^{2}\left(1+4 \pi p_{0} B_{z}^{-2} \cos ^{2} \theta\right)\right. \\
& \left.-4 \pi c^{-1}\left(\partial j_{y} / \partial A_{0}\right)\right\} A_{1 y} \\
= & -j^{\text {res }}\left(z, \theta, A_{1 y}, t\right)
\end{aligned}
$$

Because the resonant current density $j^{\text {res }} \sim \int_{0}^{t} K\left(t-t^{\prime}\right)$ $A_{1}\left(t^{\prime}\right) d t^{\prime}$ depends on time, Eqs. (7) and (8) could be considered as the evolutionary ones

$\left(\bar{D} A_{1}(z, t)=S(z) \int_{0}^{t} K\left(t-t^{\prime}\right) A_{1}\left(z, t^{\prime}\right) d t^{\prime}\right.$,

$\bar{D}=d^{2} / d z^{2}-D_{0}(z)$ is the differential operator) and could be solved by the method of finite elements (Lapenta and Brackbill, 1997; Daughton, 1999). For this purpose, one could use the basis of Hermite polynomials' spatial functions $H_{n}(z)(n=0,1,2, \ldots)$ and then obtain the following form of perturbed vector potential: $A_{1}=\sum_{n} H_{n}(z) \psi_{n}(t)$. The corresponding system of integral equations is the following: $\quad T_{n m} \psi_{n}(t)=S_{n m} \int_{0}^{t} K\left(t-t^{\prime}\right) \psi_{n}\left(t^{\prime}\right) d t^{\prime} \quad$ (here $\left.T_{n m}=\int_{-\infty}^{\infty} H_{m} \bar{D} H_{n} d z, \quad S_{n m}=\int_{-\infty}^{\infty} H_{m} S(z) H_{n} d z\right) . \quad$ Finally, from this system of equations, we obtained the corresponding eigenfrequencies and growth rates of wave perturbations as functions of TCS parameters, wavenumbers $k$ and propagation angles $\theta$ (Fig. 1).

The resulting growth rates for both kinds of polarization depending on the angles $\theta$ are shown in Fig. 2. As one can see, both symmetric polarization modes have equal positive 


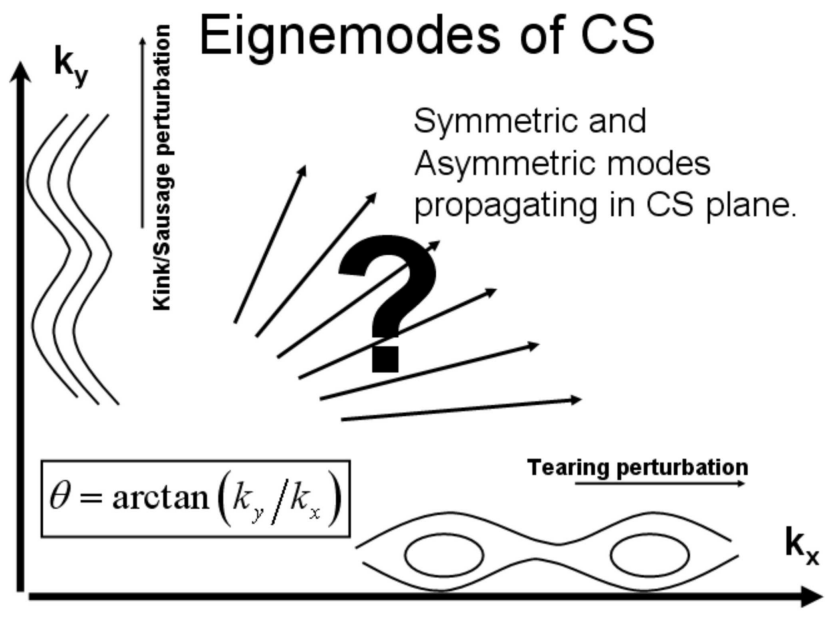

Fig. 1. Sketch of eigenmodes in the CS.

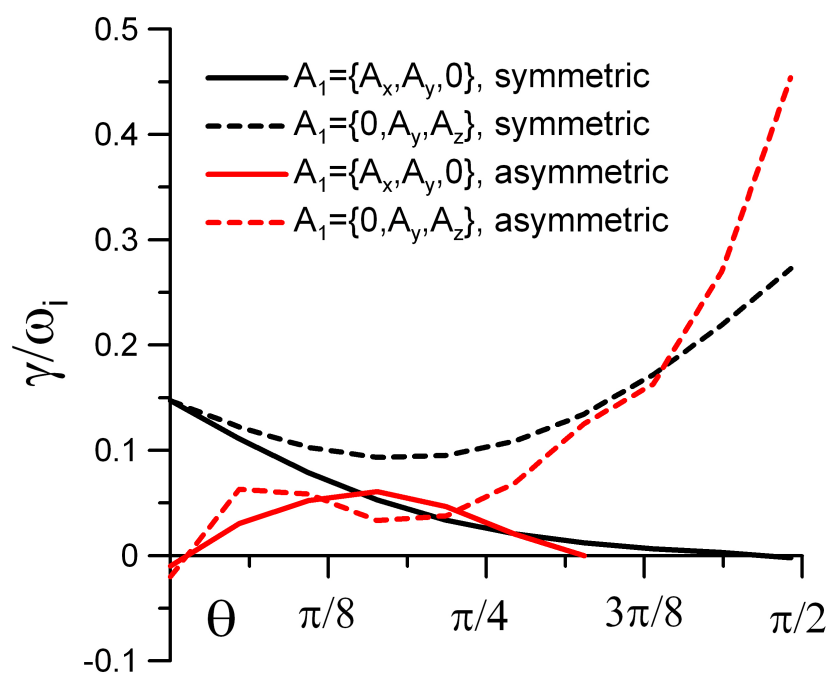

Fig. 2. Growth rate as a function of angle $\theta$ for two polarizations. The parameters have the following values: $\tau=3, L_{C S}=0.8 \rho_{i}$, $k L_{C S}=0.3, b_{n}=0.1$.

values of growth rate in the case of tearing instability $(\theta=0)$, but asymmetric modes are suppressed when $\theta \rightarrow 0$. When the propagation angle $\theta$ increases, the perturbation with polarization $\boldsymbol{A}_{1}=A_{1 x} \boldsymbol{e}_{x}+A_{1 y} \boldsymbol{e}_{y}$ is suppressed. However, with another polarization, $\boldsymbol{A}_{1}=A_{1 y} \boldsymbol{e}_{y}+A_{1 z} \boldsymbol{e}_{z}$ becomes correspondingly sausage or kink instabilities at $\theta=\pi / 2$. In the range $\theta \sim \pi / 2$, the growth rate of perturbation becomes larger than the one of the tearing mode $(\theta \sim 0)$. Thus, the development of perturbations propagating along the current ("Y" direction) are more probable than the development of a tearing mode (i.e. waves moving along the " $\mathrm{X}$ " direction). Also, the asymmetric mode perturbations at $\theta=\pi / 2$ have larger growth rates than the symmetric one; this is similar to the behaviour obtained in Harris CSs (Daughton, 1999).

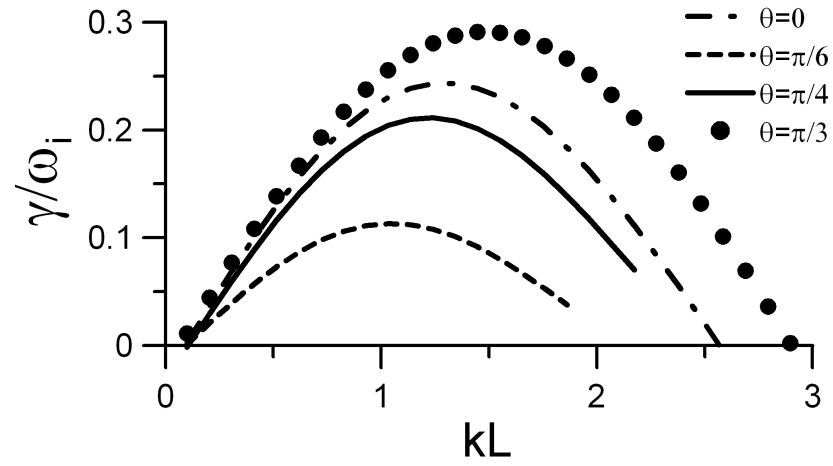

Fig. 3. Growth rate as a function of the magnitude of wavenumber $k$ for polarizations $\boldsymbol{A}=A_{y} \boldsymbol{e}_{y}+A_{z} \boldsymbol{e}_{z}$. The parameters have the following values: $\tau=3, L_{C S}=0.6 \rho_{i}, b_{n}=0.1$.

Figure 3 demonstrates the growth rate of the symmetric mode with polarization $\boldsymbol{A}_{1}=A_{1 y} \boldsymbol{e}_{y}+A_{1 z} \boldsymbol{e}_{z}$ as a function of wavenumber $k$. As one can see, the range of wavenumbers with positive values of growth rate is wider than the one in the classical Harris CS (Daughton, 1999). The maximum value of the growth rate for different values of propagation angles belongs to segment $k L_{C S} \in[0.8,1.8]$. The real part of the frequency in this region of wavenumbers belongs to the value range $\omega \in[0.01,0.035] \omega_{i}$ (which is common to both symmetric and asymmetric modes). These values are much smaller than in the "classical" case of thin Harris CSs (Lapenta and Brackbill, 1997; Daughton, 1999) because the real part of the frequency is proportional to the velocity of diamagnetic drifts $\omega \approx k_{y} v_{D M}=k v_{D M} \sin \theta$, where $v_{D M}$ is the velocity of the diamagnetic drift (Baumjohann and Treumann, 1996). In the Harris CS, where most of the current is supported by diamagnetic particle drifts $v_{D M} \sim d n / d z$, the plasma density gradients are much larger than in the anisotropic CS with few embedded layers, if the thicknesses of the Harris CS and the anisotropic one are close.

\section{Experimental data}

Oscillations of the magnetotail current sheet (flappings) were regularly observed by the multispacecraft Cluster mission (Sergeev et al., 2004; Runov et al., 2006; Petrukovich et al., 2006). To compare with the predictions of our theory a vast variety of such flapping motions, we select a group of fast quasi-periodic oscillations (with periods of not longer than $3 \mathrm{~min}$ ). The set of fourteen events from the years 2001, 2003 and 2004 contains 160 fast crossings (Table 1). The magnetic field and the plasma density (measured by CODIF at C4) data were taken from the CAA database.

Such oscillations form a specific subclass of flapping motions. They have a relatively high frequency (about $0.01 \mathrm{~Hz}$ ) and often a rather high degree of monochromaticity. The 


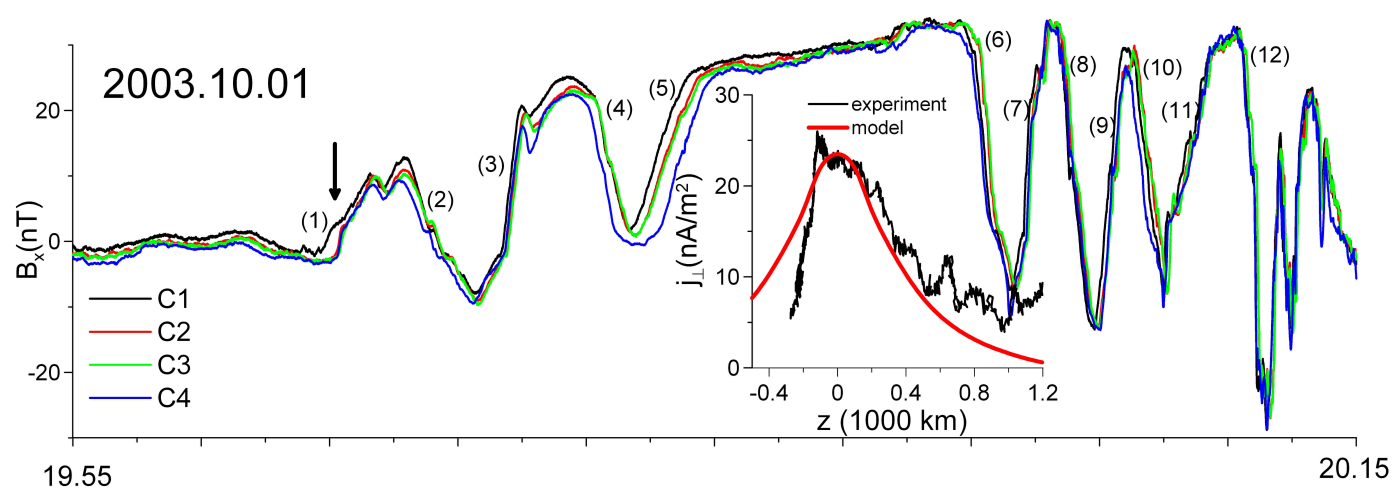

Fig. 4. Component of magnetic field $B_{x}$ observed by four spacecraft and comparison of the current density profile with that of our TCS model (in the insert). Individual crossings are marked by numbers.

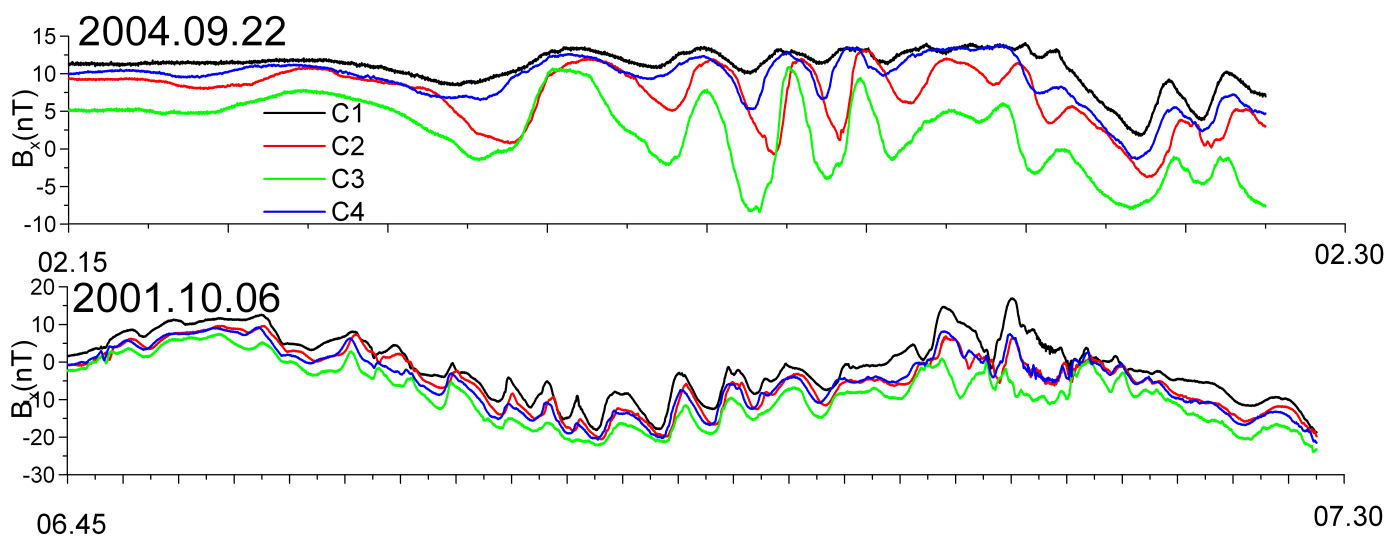

Fig. 5. Two other examples of wavy events (see the text for details).

plasma sheet has values of $B_{z}$ and $B_{y}$ within $5 \mathrm{nT}$ and most often around $1-2 \mathrm{nT}$. This subclass is distinctly different from, for example, the slower and steeper (up to 80degree tilts) oscillations typical of situations with a large $B_{z}$ (Petrukovich et al., 2006). In this sense, the statistics of Sergeev et al. (2004) and Runov et al. (2006), including all the fast crossings during 2001, are more general and contain some of our examples. On the other hand, our selection includes the data from the years 2003 and 2004 and some additional events from 2001 missed by Runov et al. (2006) due to formal reasons (for example, due to the absence of the neutral plane crossings).

As a first example, we choose one event previously analysed thoroughly by Nakamura et al. (2006). Figure 4 demonstrates a magnetic field $B_{x}$, typical of our statistics. The twelve individual crossings forming a quasi-periodic sequence are marked by number. The two main frequencies are $\nu_{1} \approx 0.06 \mathrm{~Hz}$ and $\nu_{2} \approx 0.0125 \mathrm{~Hz}$ (found with the help of Fourier analysis). The current density profile of a sample crossing (\#1) is quite consistent with the prediction of our anisotropic TCS model (insert in Fig. 4). The detailed comparison of the profiles was performed by Artemyev et al. (2008).
For a better illustration of the selected events, two additional examples are shown in Fig. 5. The event of 22 September 2004 helps us to understand the vertical spatial scale of oscillations, which is of the order of a Cluster tetrahedron size $\sim 1000 \mathrm{~km}$. The spacecraft outside the embedded sheet at the level of $B_{x} \sim 15 \mathrm{nT}$ practically does not observe any oscillation, but Cluster 3 located near the neutral plane observes waves with a magnitude of about $10 \mathrm{nT}$ and a period of about one minute. Oscillations occur in a quiet thin sheet after the period of thinning and stretching (Petrukovich et al., 2007) and immediately before the start of the dipolarization. This interesting coincidence between the appearance of such oscillations and sheet disruption will be studied in following publications.

The event from 6 October 2001 exhibits oscillations with rather small amplitudes, observed at a distance by Cluster from the neutral plane. This event therefore was not included in Runov et al.'s statistics (2006), but otherwise the properties of such oscillations are quite typical.

Events similar to our statistics have also been found in the 2005-2007 Cluster data. However, for this period, it was impossible to determine wave directions and spatial scales because of the large spacecraft separation $\left(\sim 1.5 R_{E}\right)$. 

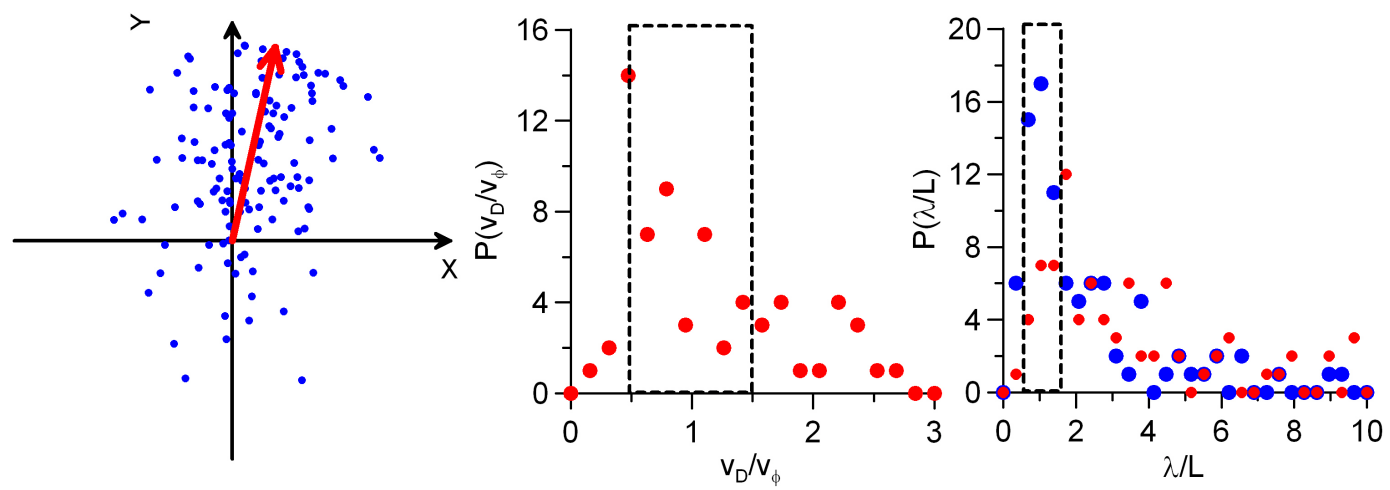

Fig. 6. Statistics of 160 fast crossings. Left: directions of normal vector projections to the $X Y$ plane for each CS crossing. Centre: the histogram of values $v_{D} / v_{\phi}$. Right: the histogram of values $\lambda / L$ for two frequencies. The blue color denotes the higher frequency and the red color - the smaller one (see text for details). Dashed boxes denote regions roughly corresponding to the theory.

Table 1. Fourteen events with fast monochromatic flapping motions.

\begin{tabular}{clrrr}
\hline$n$ & date & $<X>$ & $\begin{array}{c}<Y_{\mathrm{gsm}}> \\
(1000 \mathrm{~km})\end{array}$ & $<Z_{\mathrm{gsm}}>$ \\
\hline 1 & 17 Aug 2001: 16:29-16:51 & -117 & -30 & 1.8 \\
2 & 26 Sep 2001: 22:19-22:49 & -114 & 46 & -3 \\
3 & 1 Oct 2001: 09:30-10:00 & -104 & 51 & 4.5 \\
4 & 6 Oct 2001: 06:45-07:30 & -104 & 60 & -9.5 \\
5 & 8 Oct 2001: 13:00-13:32 & -99 & 62 & -0.1 \\
6 & 8 Oct 2001: 13:30-13:45 & -99 & 62 & -0.8 \\
7 & 11 Oct 2001: 03:25-03:45 & -100 & 70 & -12.2 \\
8 & 24 Aug 2003: 18:37-18:47 & -107 & -24 & 20.5 \\
9 & 1 Oct 2003: 20:00-20:15 & -103 & 44 & -0.4 \\
10 & 1 Nov 2003: 13:45-13:55 & -63 & 77 & -9 \\
11 & 22 Sep 2004: 02:15-02:30 & 113 & 27 & -11 \\
12 & 15 Oct 2004: 12:35-12:45 & -78 & 52 & 2.2 \\
13 & 15 Oct 2004: 12:45-13:00 & -80 & 52 & 1.8 \\
14 & 15 Oct 2004: 14:30-14:50 & -84 & 56 & -3.8 \\
\hline
\end{tabular}

We obtain the values of sheet velocity along the normal $\boldsymbol{v}_{n}$ and the normal direction for each crossing as well as the magnitude of drift velocity $\boldsymbol{v}_{D}=\left(\boldsymbol{j} / e n_{p}\right)_{B_{x}=0}$ averaged over the time of the given crossing ( $\boldsymbol{j}$ is the current density defined with the curlometer technique and $\boldsymbol{v}_{n}$ can be obtained as $|\Delta \boldsymbol{r} / \Delta t|$ where $\Delta t$ is the time shift between the spacecraft observations and $\Delta \boldsymbol{r}$ is the distance between the spacecraft). We assume that the phase velocity of wave $\boldsymbol{v}_{\phi}$ is of the order of $\boldsymbol{v}_{n}$. The estimation of the wavelength of these oscillations is $\lambda=\left|\boldsymbol{v}_{\varphi}\right| / \nu$ (where $v$ - frequency of oscillation). Another spatial scale - sheet thickness - was estimated as $L=B_{\text {ext }} /|\boldsymbol{j}|_{B_{x}=0}$. Here, $B_{\text {ext }}^{2} / 8 \pi=1.17 T_{p} n_{p}$ (the factor 1.17 is used to account for the electron contribution to the total pressure).
Finally, the values of sheet velocity $\boldsymbol{v}_{\varphi}$ and drift velocity $\boldsymbol{v}_{D}$, the estimates of wavelength and sheet thickness and the directions of propagation normal in the $X Y$ plane for all 160 crossings are summarized in Fig. 6. In order to access the spread in our estimates, we work out the wavelengths for the two frequencies corresponding to the two main maxima of the spectral power. The dominant direction of wave propagation in the $X Y$ plans is almost along the current $(Y)$. The wave phase velocities are of the order of drift velocity. The wavelengths of the oscillations are of the order of thickness of CS. Theoretical values obtained in our linear analysis of drift instabilities are shown by boxes (Fig. 6) and are quite consistent with the observations.

\section{Discussion and conclusions}

The linear stability analysis of low frequency wave modes propagating in the thin current sheet shows that unstable modes can have two different polarizations (with the perturbed vector potentials $\boldsymbol{A}_{1}=A_{1 y} \boldsymbol{e}_{y}+A_{1 z} \boldsymbol{e}_{z}$ and $\left.\boldsymbol{A}_{1}=A_{1 y} \boldsymbol{e}_{y}+A_{1 x} \boldsymbol{e}_{x}\right)$. These modes can also be symmetrical and asymmetrical. Perturbation modes such as the classical sausage and kink modes might exist in the counter-phase regime, i.e. if one of them is suppressed at $\theta \sim \pi / 2$, the second one reaches a maximum growth rate at this angle. The growth rate of wave modes is obtained as a function of both the amplitude of the wave vector and its direction. Contrary to the tearing stability of the Harris CS with $B_{z} \neq 0$ (Pellat et al., 1991), in the anisotropic TCS, tearing has a positive growth rate (see the detailed discussion by Zelenyi et al., 2008).

Both the tearing and kink/sausage modes are the limiting cases of a more general class of oblique CS eigenmodes, which might propagate in a CS at arbitrary angles and might become unstable in a large area of parameters. Conceptually, we would like to stress that even for eigenmode class perturbations observed within a CS (non-eigenmode transient 
events could also exist in magnetotail), their configuration could actually be a mix of differing polarizations and symmetry.

A special class of fast quasi-periodic flapping motions of CSs, observed by Cluster during 2001 to 2004, has properties quite consistent with these theory predictions: the directions of the observed flapping motions generally correspond to the current direction, while the typical velocity of the motion is comparable to the drift velocity of the CS. The frequencies and scales of the oscillations are also in agreement with the theory.

Since the direction of plasma drift velocity in any CS coincides with the direction of the current, these low frequency eigenmodes should possess properties of drift waves. This feature is very similar both in the Harris CS model (Lapenta and Brackbill, 1997; Buechner and Kuska, 1999; Daughton, 1999) and in the model of anisotropic TCS, but the latter predicts the value of a wave frequency at least a factor of 4-5 smaller than in Harris-like models. The broad variety of Cluster flapping events was interpreted by a number of models, including ones with a non-drift nature (Golovchanskaya et al., 2005; Erkaev et al., 2008; Saito et al., 2008). However, we show that at least this subclass of wavy flapping can be described by drift eigenmodes.

Acknowledgements. This work was supported in part by the RF Presidential Program for State Support of Leading Scientific Schools (project no. NSh-472.2008.2) and the Russian Foundation for Basic Research (project nos. 08-02-00407, 06-05-90631 and 0702-00319).

Editor in Chief W. Kofman thanks C. Cully and another anonymous referee for their help in evaluating this paper.

\section{References}

Artemyev, A. V., Petrukovich, A. A., Zelenyi, L. M., Malova, H. V., Popov, V. Y., Nakamura, R., Runov, A., and Apatenkov, S.: Comparison of multi-point measurements of current sheet structure and analytical models, Ann. Geophys., 26, 2749-2758, 2008,

http://www.ann-geophys.net/26/2749/2008/.

Baumjohann, W. and Treumann, R. A.: Basic space plasma physics, Imperial College Press, London, 1996.

Baumjohann, W., Roux, A., Le Contel, O., Nakamura, R., Birn, J., Hoshino, M., Lui, A. T. Y., Owen, C. J., Sauvaud, J.-A., Vaivads, A., Fontaine, D., and Runov, A.: Dynamics of thin current sheets: Cluster observations, Ann. Geophys., 25, 1365-1389, 2007, http://www.ann-geophys.net/25/1365/2007/.

Büchner, J. and Kuska, J.-P.: Sausage mode instability of thin current sheets as a cause of magnetospheric substorms, Ann. Geophys., 17, 604-612, 1999, http://www.ann-geophys.net/17/604/1999/.

Coppi, B., Laval, G., and Pellat, R.: Dynamics of the geomagnetic tail, Phys. Rev. Letters, 16(26), 1207-1210, 1966.

Daughton, W.: The unstable eigenmodes of a neutral sheet, Phys. Plasmas, 6(4), 1329-1343, 1999.

Dobrowolny, M.: Instability of a neutral sheet, Nuovo Cimento, LV B(2), 427-441, 1968.
Erkaev, N. V., Semenov, V. S., and Biernat, H. K.: Magnetic double gradient mechanism for flapping oscillations of a current sheet, Geophys. Res. Lett., 35(2), L02111, doi:10.1029/2007GL032277, 2008.

Galeev, A. A. and Zelenyi, L. M.: Tearing instability in plasma configurations, Zhurnal Eksperimental'noi i Teoreticheskoi Fiziki, 70(6), 2133-2151, 1976 (in Russian).

Golovchanskaya, I. V. and Maltsev, Y. P.: On the identification of plasma sheet flapping waves observed by Cluster, Geophys. Res. Lett., 32, L02102, doi:10.1029/2004GL021552, 2005.

Harris, E. G.: On a plasma sheet separating regions of oppositely directed magnetic field, Nuovo Cimento, 23, 115, 1962.

Kan, J. R.: On the structure of the magnetotail current sheet, J. Geophys. Res., 78, 3773-3781, 1973.

Lapenta, G. and Brackbill, J. U.: A kinetic theory for the drift-kink instability, J. Geophys. Res., 102(A12), 27099-27108, 1997.

Nakamura, R., Baumjohann, W., Runov, A., and Asano, Y.: Thin current sheets in the magnetotail observed by Cluster, Space Sci. Rev., 122, 29-38, 2006.

Pellat, R., Coroniti, F. V., and Pritchett, P. L.: Does ion tearing exist? Geophys. Res. Lett., 18, 143-146, 1991.

Petrukovich, A. A., Zhang, T. 1., Baumjohann, W., Nakamura, R., Runov, A., Balogh, A., and Carr, C.: Oscillatory magnetic flux tube slippage in the plasma sheet, Ann. Geophys., 24, 16951704, 2006,

http://www.ann-geophys.net/24/1695/2006/.

Petrukovich, A. A., Baumjohann, W., Nakamura, R., Runov, A., Balogh, A., and Rème, H.: Thinning and stretching of the plasma sheet, J. Geophys. Res., 112(A10), A10213, doi:10.1029/2007JA012349, 2007.

Runov, A., Sergeev, V. A., Nakamura, R., Baumjohann, W., Apatenkov, S., Asano, Y., Takada, T., Volwerk, M., Vörös, Z., Zhang, T. L., Sauvaud, J.-A., Rème, H., and Balogh, A.: Local structure of the magnetotail current sheet: 2001 Cluster observations, Ann. Geophys., 24, 247-262, 2006, http://www.ann-geophys.net/24/247/2006/.

Saito, M. H., Miyashita, Y., Fujimoto, M., Shinohara, I., Saito, Y., Liou, K., and Mukai, T.: Ballooning mode waves prior to substorm-associated dipolarizations: Geotail observations, Geophys. Res. Lett., 35(7), L07103, doi:10.1029/2008GL033269, 2008.

Schindler, K.: A theory of the substorm mechanism, J. Geophys. Res., 79(19), 2803-2810, 1974.

Schindler, K.: Physics of space plasma activity, Cambridge University Press, ISBN: 9780521858977, doi:10.2277/0521858976, November 2006.

Sergeev, V., Runov, A., Baumjohann, W., Nakamura, R., Zhang, T. L., Balogh, A., Louarnd, P., Sauvaud, J.-A., and Reme, H.: Orientation and propagation of current sheet oscillations, Geophys. Res. Lett., 31(5), L05807, doi:10.1029/2003GL019346, 2004.

Silin, I., Büchner, J., and Zelenyi., L.: Instabilities of collisionless current sheets: theory and simulations, Phys. Plasmas, 9(4), 1104-1112, 2002.

Sitnov, M. I., Malova, H. V., and Lui, A. T. Y.: Quasi-neutral sheet tearing instability induced by electron preferential acceleration from stochasticity, J. Geophys. Res., 102(A1), 163-174, 1997.

Sitnov, M. I., Swisdak, M., Guzdar, P. N., and Runov, A.: Structure and dynamics of a new class of thin current sheets, J. Geophys. Res., 111(A8), A08204, doi:10.1029/2005JA011517, 2006. 
Volwerk, M., Glassmeier, K.-H., Runov, A., Baumjohann, W., Nakamura, R., Zhang, T. L., Klecker, B., Balogh, A., and Rème, H.: Kink mode oscillation of the current sheet, Geophys. Res. Lett., 30(6), 1320, doi:10.1029/2002GL016467, 2003.

Zelenyi, L. M., Malova, H. V., Popov, V. Yu., Delcourt, D., and Sharma, A. S.: Nonlinear equilibrium structure of thin currents sheets: influence of electron pressure anisotropy, Nonlin. Processes Geophys., 11, 579-587, 2004,

http://www.nonlin-processes-geophys.net/11/579/2004/.
Zelenyi, L. M., Malova, H. V., Popov, V. Y., Delcourt, D. C., Ganushkina, N. Y., and Sharma, A. S.: "Matreshka" model of multilayered current sheet, Geophys. Res. Lett., 33(5), L05105, doi:10.1029/2005GL025117, 2006.

Zelenyi, L., Artemyev, A., Malova, H., and Popov, V.: Marginal stability of thin current sheets in the Earth's magnetotail, J. Atmos. Solar-Terr. Phys., 70, 325-333, 2008. 